\title{
On the reversibility of ECAs with fully asynchronous updating: the recurrence point of view (draft paper)
}

\author{
Nazim Fatès*, Biswanath Sethi†and Sukanta Das ${ }^{\ddagger}$
}

August 3, 2017

\begin{abstract}
The reversibility of classical cellular automata is now a well-studied topic but what is reversibility when the evolution of the system is stochastic? In this context, we study a particular form of reversibility: the possibility of returning infinitely often to the initial condition after a random number of time steps. This corresponds to the recurrence property of the system.

We analyse this property for the 256 elementary cellular automata with a finite size and a fully asynchronous updating, that is, we update only one cell, randomly chosen, at each time step. We show that there are 46 recurrent rules which almost surely come back to their initial condition. We analyse the structure of the communication graph of the system and find that the number of the communication classes may have different scaling laws, depending on the active transitions of the rules (those for which the state of the cell is modified when an update occurs).
\end{abstract}

\footnotetext{
*Inria Nancy, Université de Lorraine, CNRS, LORIA, F-54000, France, nazim.fates@loria.fr

†Indira Gandhi Institute of Technology, Sarang, Odisha, India

¥Indian Institute of Engineering Science and Technology, Shibpur, West Bengal, India
} 


\section{Introduction}

The question of reversibility is an important topic in the study of discrete dynamical systems because it is intimately linked to the question of time. In the context of cellular automata, a great amount of efforts have been deployed to understand how the local rule which defines the interaction between cells may generate a global behaviour which is reversible [Mor08]. However, the majority of the results concern deterministic cellular automata where reversibility has been interpreted as "backward determinism" [AP72, Ric72, Tof77], that is, the fact the each global state of the system, the configuration, has only a unique predecessor. In the specific case of finite cellular automata, this implies that the system will necessarily return to its initial state after some finite number of iterations.

The question can then be raised to know what is the equivalent of reversibility when the evolution of the cellular automaton is submitted to a form of noise. The non-determinism of the evolution implies that each configuration may have several successors. Consequently, the one-to-one correspondence between a configuration and its image can no longer be taken as a criterion of reversibility. Instead, the criterion which consists in looking if the system comes back to its initial condition may lead to interesting classifications of rules, depending on the conditions under which this "eternal return" phenomenon happens.

In this contribution, we consider the case of elementary cellular automata with fully asynchronous update. In other words, we study finite-size systems, with a local rule that involves nearest-neighbour interaction and binary states, and at each time step, we update one cell chosen randomly and uniformly in the set of cells.

So far, only a few results on the reversibility of asynchronous cellular automata have been obtained. In the case where one is allowed to choose freely the order of update of the cells, it was shown that there are some rules which allow one to return to the initial condition and some that do not always allow this [DSS12, SMD12]. With a different perspective, Worsch and Wacker examined how to construct an "inverse" rule, in the sense that its transition graph would be the "inverse" of the transition graph of the original inverse [WW13]. The design of asynchronous circuits with reversible gates is also a current important topic of research (see e.g. Ref. $\left[\mathrm{PLC}^{+} 13, \mathrm{LPC}^{+} 16\right]$ and references therein).

In a previous work, we analysed the set of elementary cellular automata with the aim of knowing in which case a cellular automaton may not return to its initial condition [SFD14]. This resulted in the identification of the irreversible rules in the sense that such rules would not always return to their initial condition: out of the 256 rules, there are 132 strongly irreversible rules, which possess at least configuration that is not reachable from itself, and 78 rules for which the system would return to its initial configuration a finite number of times and then stay away from it for ever. It was conjectured that the rest of the rules would be recurrent in the sense that that would always return to their

initial condition an infinite number of times. Our goal is to formally prove this recurrence property for the set of the remaining 46 candidate rules. 
Table 1: Table showing the transitions and their labels.

\begin{tabular}{cccc}
\hline A & B & C & D \\
000 & 001 & 100 & 101 \\
\hline \hline 010 & 011 & 110 & 111 \\
E & F & G & H \\
\hline
\end{tabular}

\section{Notations}

\section{1 basis}

We consider one-dimensional binary cellular automata with periodic boundary conditions. The cells are arranged in a ring and the set of indices that represent each cell is denoted by $\mathcal{L}=\mathbb{Z} / n \mathbb{Z}$, where $n>1$ is the number of cells. We exclude the case $n=1$ to avoid having a cell connected only to itself. At each time step $t \in \mathbb{N}$, a cell is assigned a state in $\{0,1\}$. The collection of all states at given time is called a configuration and the set of configurations is denoted by $\mathcal{E}_{n}=\{0,1\}^{\mathcal{L}}$.

We will often assimilate a configuration to a circular word and we use $|x|_{w}$ to denote the number of occurrences of the sub-word $w \in Q^{*}$ in the circular word formed by the states of $x$. A 1-region (resp. 0-region) corresponds to a maximal set of contiguous cells with the state 1 (resp. 0). Note that for a configuration different from $\mathbf{0}$ and $\mathbf{1}$, the number of 0 -regions and 1-regions are equal.

An elementary cellular automaton (ECA) is a function $f:\{0,1\}^{3} \rightarrow\{0,1\}$. A rule $f$ describes the local transformations $(x, y, z) \rightarrow f(x, y, z)$ with $(x, y, z) \in$ $\{0,1\}^{3}$; we call each quadruplet $(x, y, z, f(x, y, z))$, a transition. We say that a transition is active if it changes the state of a cell $(f(x, y, z) \neq y)$; it is passive otherwise. The decimal code associated to $f$ is the number: $W(f)=$ $f(0,0,0) \cdot 2^{0}+f(0,0,1) \cdot 2^{1}+\cdots+f(0,0,1) \cdot 2^{7}$.

Since its introduction by Wolfram, this code has become a standard way to identify the ECAs. We will also use another code, called the transition code, to identify an ECA. This code consists of a concatenation of letters in $\{A, \ldots, H\}$, where each letter denotes an active transition of the rule. The association of a letter to a transition follows the mapping of Table 1.

In the following, a rule will be denoted both by its decimal code and its transition code. For instance, majority rule is 232 :DE (only 101 and 010 are active; this corresponds to RMT 5 and 2 in the notation used by Das et al. [SMD12]). The transition code of special rule that has no active transition is 204:I, where $I$ stands for identity.

Given a local rule $f$ and a ring size $n$, we define the global transition rule with fully asynchronous updating as $F: \mathcal{E}_{n} \times \mathcal{L} \rightarrow \mathcal{E}_{n}$ which maps a configuration $x \in \mathcal{E}_{n}$ and a cell to update $c \in \mathcal{L}$ to the configuration $y$ such that:

$$
\forall i \in \mathcal{L}, y_{i}= \begin{cases}f\left(x_{i-1}, x_{i}, x_{i+1}\right) & \text { if } i=c, \\ x_{i} & \text { otherwise }\end{cases}
$$


Starting from an initial condition $x$, the evolution of the system can thus be described by the Markov chain $\left(X^{t}\right)_{t \in \mathbb{N}}$, where the random variables $X^{t}$ are obtained recursively with $X^{0}=x$ (with prob. 1) and $X^{t+1}=F\left(X^{t}, U^{t}\right)$ where $\left(U^{t}\right)_{t \in \mathbb{N}}$ is a series of uniformly and identically distributed random variables on $\mathcal{L}$.

\subsection{Defining recurrence}

Recall that our aim is to know, given a cellular automaton $f$ and a ring size $n$, if this system always returns to its initial condition. Since the updating is stochastic (one cell is chosen randomly and uniformly at each time step), one may believe at first sight that advanced tools from probability theory are needed to answer this question. In fact, because we are dealing with finite and memoryless systems, we only need some simple notions form the Markov chain theory. We now introduce these tools.

Definition 1. For two arbitrary configurations $x, y \in \mathcal{E}_{n}$, we say that $y$ is a successor of $x$ if there exists an update $u \in \mathcal{L}$ such that $F(x, u)=y$. For $x \in \mathcal{E}_{n}$, we denote by $\operatorname{succ}(x)=\{F(x, u), u \in \mathcal{L}\}$ the set of successors of $x$ and $\operatorname{succ}(X)=\cup_{x \in X} \operatorname{succ}(x)$ the set of successors of a set $X \subseteq \mathcal{E}_{n}$.

By iterating the successor relation, we obtain a new relation, "being reachable".

Definition 2. For two arbitrary configurations $x, y \in \mathcal{E}_{n}$, we say that $y$ is reachable from $x$ if $y$ can be obtained from $x$ by following a sequence of successor relations, that is, $\exists\left(x^{i}\right), i \in\{0, \ldots, k\}$ such that $x^{0}=x, x^{k}=y$ and $x^{i+1} \in$ $\operatorname{succ}\left(x^{i}\right)$ for $i \in\{0, \ldots, k-1\}$.

We denote by $x \mapsto y$ that $y$ is reachable from $x$ and the sequence of successors that leads from $x$ to $y$ is denoted by $\llbracket x=x^{0}, x^{1}, \ldots, x^{k}=y \rrbracket$. We call this sequence a chain of successors, or simply a chain.

We can now define the recurrence property:

Definition 3. A configuration $x \in \mathcal{E}_{n}$ is recurrent if for every configuration $y$ that is reachable from $x, x$ is also reachable from $y$. A configuration that is not recurrent is transient.

Intuitively, a transient configuration is such that there exists a particular sequence of updates which brings the system to a configuration from which it will never be possible to return back to the initial configuration.

More generally, if $y$ is reachable from $x$ and $x$ is reachable from $y$, we say that $x$ and $y$ communicate. By convention, all states communicate with themselves. Clearly, the relationship "communicate" is an equivalence relation; this relation partitions the set of configurations into communication classes. Consequently, two major behaviours exist: for the transient configurations, the system remains a finite time in its communication class, then "escapes" this class and never returns back to it. In contrast, when the system starts from a recurrent 
configuration, it remains in the communication class of this configuration for ever.

Definition 4. A rule is recurrent for size $n$ if each configuration of $\mathcal{E}_{n}$ is recurrent; otherwise, it is irreversible (for this size). A rule is recurrent if it is recurrent for all the sizes $n>1$.

The set of the 256 ECA can be partitioned into recurrent and irreversible rules according to the following theorem:

Theorem 1. Among the 88 minimal ECA rules, the following 16 rules are re-

\begin{tabular}{llll|} 
current: & \multicolumn{4}{c|}{} \\
\hline $35: A B D E F G H$ & $38: B D F G H$ & $43: A B D E G H$ & $46: B D G H$ \\
$51: A B C D E F G H^{*}$ & $54: B C D F G H^{*}$ & $57: A C D E G H^{*}$ & $60: C D G H^{*}$ \\
$62: B C D G H$ & $105: A D E H^{*}$ & $108: D H^{*}$ & $134: B F G$ \\
$142: B G$ & $150: B C F G^{*}$ & $156: C G$ & $204: I^{*}$ \\
\hline
\end{tabular}

The rules 33:ADEFGH and 41:ADEGH are recurrent for $n \neq 3$.

The rules with an asterisk are directly recurrent (see below).

\subsection{Direct recurrence}

Definition 5. We say that a rule has the property of direct recurrence if for any two configurations $x, y \in \mathcal{E}_{n}$, if $y$ is a successor of $x$, then $x$ is also a successor of y. Formally: $\forall x \in \mathcal{E}_{n}, x \in \operatorname{succ}(\operatorname{succ}(x))$.

Proposition 1. A rule that is directly recurrent is recurrent.

Proof. Consider a directly recurrent rule and assume that $x$ and $y$ are two configurations such that $x \longmapsto y$ with the chain of successors $\llbracket x^{0}=x, \ldots, x^{k}=y \rrbracket$. The direct recurrence property gives $x^{i} \in \operatorname{succ}\left(x^{i+1}\right)$ for all $i \in\{0, \ldots, k-1\}$, which implies that $y \longmapsto x$ with the chain $\llbracket x^{k}=y, \ldots, x^{0}=x \rrbracket$.

Proposition 2. A rule is directly recurrent if and only if it contains 0 or 2 active transitions in each of the four sets of transitions: $\{A, E\},\{B, F\},\{C$, $\mathrm{G}\},\{\mathrm{D}, \mathrm{H}\}$.

Proof. The proof is clear by looking at Table 1 (p. 3). If the rule has no active transition, that is, if we have the identity rule, then the rule is recurrent because every point is a fixed point. Let us now assume for example that we go from $x$ to $y$ by applying transition $\mathrm{A}(000 \rightarrow 1)$. Then, on the configuration $y$, on the cell where the transition was applied, it is now possible to apply transition $\mathrm{E}(010 \rightarrow 0)$ to return to the previous configuration $x$. Symmetrically, if $\mathrm{E}$ was applied first, then it is possible to apply A to reverse the effect of the transition. The same arguments apply for the other couples of transitions $\{B, F\},\{C, G\}$, and $\{D, H\}$. Reciprocally, if a rule is directly recurrent, one of these cases apply.

We thus determine the minimal rules which are directly recurrent as: 51 : ABCDEFGH, $54:$ BCDFGH, $57:$ ACDEGH, $60: \mathrm{CDGH}, 105: \mathrm{ADEH}, 108: \mathrm{DH}, 150: \mathrm{BCFG}$, $156:$ CG, $204:$ I. 


\subsection{A lemma for determining the communication classes}

Lemma 1. For a given rule $f$ and a given ring size $n$, let $\left(E_{i}\right)_{i \in\{1, \ldots, k\}}$ be a partition of the configuration space $\mathcal{E}_{n}$ and let $\left(r_{i}\right)$ be a sequence of configurations such that $\forall i \in\{1, \ldots, k\}, r_{i} \in E_{i}$. If for each $i \in\{1, \ldots, k\}$ :

(a) Any configuration of $E_{i}$ communicates with $r_{i}$,

(b) $E_{i}$ is closed under the application of $F$ (that is, $\operatorname{succ}\left(E_{i}\right) \subseteq E_{i}$ ),

then $f$ is recurrent and its communication classes are formed by the sets $\left(E_{i}\right)$.

To verify the lemma, one simply needs to observe that for each $i \in\{1, \ldots, k\}$, the set $E_{i}$ is recurrent and maximal. Consider $x \in E_{i}$, if we take a configuration $y \in E_{i}$, then $x$ and $y$ communicate through $r_{i}$ and if we take a configuration $y$ out of $E_{i}$ than $y$ is not reachable from $x$, otherwise this would contradict the second hypothesis which stipulates that the set $E_{i}$ are closed.

Since communication classes are essentially equivalence classes, we call the configurations $\left(r_{i}\right)$ the representatives of the partition $\left(E_{i}\right)$. In our proofs, we will often show that two different representatives do not communicate, which is equivalent to the second property of the lemma.

\section{Analysis of the recurrent rules}

Let us now analyse the recurrent ECAs and their communication classes. We start with the rules that have a large number of communication classes and finish with the rules where the communication class is the whole set of configurations $\varepsilon_{n}$.

\subsection{Rules with an exponential number of communication classes}

Clearly, the identity rule 204:I is recurrent and because each configuration is a fixed point and thus constitutes its own communication class.

Proposition 3. Rules 156:CG is recurrent. The number of its communication classes $\mathcal{C}_{\mathrm{CG}}$ scales exponentially with the ring size $n$. More precisely, we have $\mathcal{C}_{\mathrm{CG}}(n) \sim \phi^{n}$, where $\phi=(1+\sqrt{5}) / 2$ is the golden ratio.

Proof. This rule is directly recurrent.

To count the number of communication classes, let us define the set of configurations which do not contain the pattern 11 as $R=\left\{x \in \mathcal{E}_{n} ; \exists i \in\right.$ $\left.\mathcal{L} ;\left(x_{i}, x_{i+1}\right)=(1,1)\right\}$. We want to show that the elements of $R$ and the fixed point 1 constitute a set of representatives. Let us take a configuration $x \notin R \cup\{\mathbf{1}\}$. This configuration necessarily contains the pattern 11 and, since it is different from $\mathbf{1}$, it contains the pattern 110 . The transition $\mathrm{G}(110 \rightarrow 0)$ is active; $x$ thus communicates with a configuration $x^{\prime}$ that has a 1-region which has been decreased by 1 (e.g. $x=0011101$ and $x^{\prime}=0011001$ ). By transitivity 


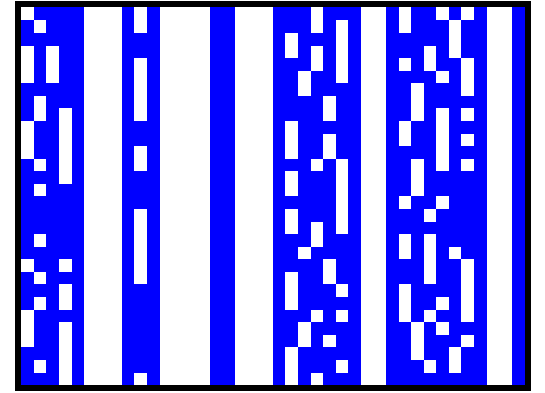

ECA 108:DH

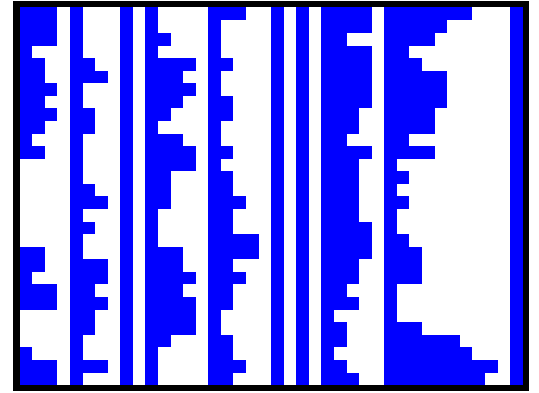

ECA 156:CG

Figure 1: Space-time diagrams of two ECA rules with an exponential number of communication classes. Blue and white squares represents cells in state 0 and 1, respectively. Time goes from bottom to top. Each row represents the state of the system after $n$ random updates of cells. We have here $n=40$ and the evolution is shown during $30 \cdot n$ time steps. This convention is kept.

$x$ communicates with $x^{\prime \prime} \in R$ which is such that all the 1-regions of $x$ have been reduced to a size 1 (e.g. $x^{\prime \prime}=0010001$ ).

Moreover, if we take two different configurations in $R$, they do not communicate. Indeed, notice that the transitions $C$ and $G$ do not modify the positions of the 01-frontiers (see Fig. 1). The set $R \cup\{\mathbf{1}\}$ is thus a set of representatives and we have $\mathcal{C}_{\mathrm{CG}}(n)=\operatorname{card} R+1$.

Now, it is well-known that the number $f_{n}$ of binary words of size $n$ that do not contain the pattern 11 follows the Fibonacci sequence, defined by: $f_{n}=$ $f_{n-1}+f_{n-2}$ with $f_{1}=2$ and $f_{2}=3$. This relationship can be proven with a recurrence on $n$ by counting the number of words which end with a 0 or a 1 , respectively. In order to obtain the size of $R$, we need to take into account the circularity of the configuration and thus to suppress the words of length $n \geq 3$ which start with a 1 and end with a 1 . The number $\delta_{n}$ of such words also verifies $\delta_{n}=\delta_{n-1}+\delta_{n-2}$ with $\delta_{3}=1$ (101) and $\delta_{4}=1$ (1001). With the convention $\delta_{2}=0, \delta_{3}=1$, for $n \geq 2$, the number of classes of communication of rule CG reads $\mathcal{C}_{C G}(n)=\left(f_{n}-\delta_{n}\right)+1$, which implies that it obeys the recursive equation $\mathcal{C}_{C G}(n)=\mathcal{C}_{C G}(n-1)+\mathcal{C}_{C G}(n-2)-1$ with $\mathcal{C}(1)=2$ and $\mathcal{C}(2)=4$. As a result, we obtain that $\mathcal{C}_{\mathrm{CG}}(n) \sim \phi^{n}$, where $\phi=(1+\sqrt{5}) / 2$ is the golden ratio $^{1}$.

Proposition 4. Rules 108:DH is recurrent. The number of its communication classes scales exponentially with the ring size $n$.

Proof. This rule is directly recurrent. Let us evaluate the number of its communication classes. Let $R$ be the set of configurations which do not have an isolated 0: We want to show that $R$ constitutes a set of representatives.

\footnotetext{
${ }^{1}$ The first elements of the sequence $\mathcal{C}_{\mathrm{CG}}(n)$ are $2,4,5,8,12,19,30$ and the sequence has index A001612 in the On-Line Encyclopedia of Integer Sequences (OEIS).
} 
Since transition $D(101 \rightarrow 1)$ is active, it is clear that any configuration $x$ which does not belong to $R$ communicates with a configuration $x^{\prime}$ with one less isolated 0 , and, by transitivity, with a configuration which has no isolated 0 , that is with an element of $R$. Moreover, notice that for any configuration, a 0-region whose length is strictly greater than 1 is stable. Consequently, two different configurations of $R$ cannot communicate because they necessarily have one 0-region for which they differ (otherwise they would be equal). The set $R$ is thus a set of representatives.

Let us now estimate the size of $R$ as a function of the ring size $n$. As for rule CG (see above), we can first evaluate the number $g_{n}$ of (non-circular) words which do not contain the pattern 101. By looking at the recurrence relations that govern the number of (legal) words which end with the patterns $00,01,10$, and 11 , one can prove that $\left(g_{n}\right)$ verifies the recurrence equation ${ }^{2}$ : $g_{n}=g_{n-1}+g_{n-2}+g_{n-4}$ with $g(0)=1, g(1)=2, g(2)=4$ and $g(3)=7$. One can then count the number $\delta_{n}$ of words which contain an isolated 0which is exclusively created by the circularity of the configuration. This corresponds to the words of the form $01 w 1$ and $1 w^{\prime} 10$ where $w$ and $w^{\prime}$ are binary words of length $n-3$ that do not contain an isolated 0 . By applying the same techniques as above, one can prove that $\delta_{n}$ obeys the same recurrence equation as $g_{n}$, but with different initial conditions. More precisely, we have: $\delta_{1}=0, \delta_{2}=\delta_{3}=\delta_{4}=2$ and $\delta_{n}=2 g_{n-4}$ for $n>4$.

As we have $\mathcal{C}_{D H}(n)=\operatorname{card} R=g_{n}-\delta_{n}=g_{n}-2 . g_{n-4}$, we obtain the recurrence equation: $\mathcal{C}_{D H}(n)=\mathcal{C}_{D H}(n-1)+\mathcal{C}_{D H}(n-2)+\mathcal{C}_{D H}(n-4)$ with $\mathcal{C}(1)=2, \mathcal{C}(2)=2, \mathcal{C}(1)=5, \mathfrak{C}(1)=10$. It should be noted that the case $n=1$, which was excluded from our study, does not correspond to a case where the rule is recurrent but it is here given only to initiate the recurrence ${ }^{3}$. To solve this linear recurrence equation, we need to find the roots of the polynomial $P(x)=x^{4}-x^{3}-x^{2}-1=(x+1)\left(x^{3}-2 x^{2}+x-1\right)$. Using a program, we found that the four roots of $P$ are $-1, \lambda \approx 1.75488$, and two complex conjugate solutions whose module is strictly smaller than 1 . As a result, we obtain that $\mathcal{C}_{D H}(n) \sim C . \lambda^{n}$ where $C$ is a constant which is determined to be equal to 1 (see Appendix p. 20).

\subsection{Rules with a linear number of communication classes}

Proposition 5. Rules 134:BFG, 142:BG, and 150:BCFG are recurrent. Their communication classes correspond to the fixed points and to the configurations with an equal number of 1 -regions.

The number of their communication classes is: $C C(n)= \begin{cases}3+n / 2 & \text { if } n \in 2 \mathbb{Z}, \\ 2+\lfloor n / 2\rfloor & \text { otherwise. }\end{cases}$

Proof. Let us first focus on rule BG. This rule has two fixed points: $\mathbf{0}$ and $\mathbf{1}$ and for $n$ even, it has two additional fixed points: $(01)^{\frac{n}{2}}$ and $(10)^{\frac{n}{2}}$.

\footnotetext{
${ }^{2}$ This corresponds to the sequence A005251 of the OEIS (with different initial conditions since their sequence $(a(n))_{n \in \mathbb{N}}$ is given by $\left.a(n)=g_{n-3}\right)$.

${ }^{3}$ This corresponds to the sequence A259967 of the OEIS.
} 


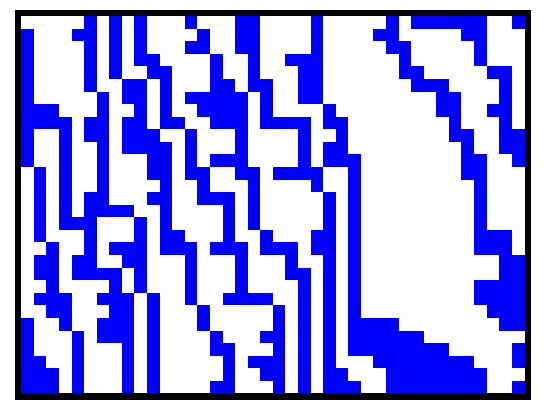

ECA $134: B F G$

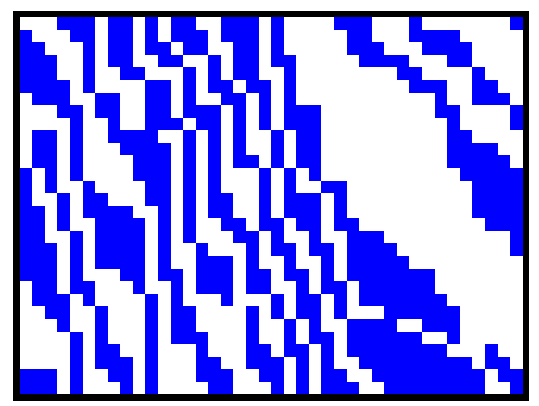

ECA 142:BG

Figure 2: Space-time diagrams of two ECA rules with a linear number of communication classes.

Table 2: Rule BG: sequence of configurations showing that the communication between an arbitrary configuration and a representative configuration. Below each configuration, we indicate which transition can be applied in each cell (a for transition $\mathrm{A}, \mathrm{b}$ for transition $\mathrm{B}$, etc.).

\begin{tabular}{cc}
\hline$x$ & $\begin{array}{l}1100111010111 \\
\text { hgcbfhgdedchh }\end{array}$ \\
\hline$x^{\prime}$ & $\begin{array}{l}0000100010100 \\
\text { aabecabedeca }\end{array}$ \\
\hline$x^{\prime \prime}$ & $\begin{array}{c}0000101010000 \\
\text { aaabededecaaa }\end{array}$ \\
\hline$s_{3}$ & $\begin{array}{l}1010100000000 \\
\text { ededecaaaaaab }\end{array}$
\end{tabular}

to a representative point

\begin{tabular}{cc}
\hline$s_{3}$ & $\begin{array}{l}1010100000000 \\
\text { ededecaaaaaab }\end{array}$ \\
\hline$y^{\prime \prime}$ & $\begin{array}{l}\text { } 000001010100 \\
\text { aaaaabededeca }\end{array}$ \\
\hline$y^{\prime}$ & $\begin{array}{l}0100000010100 \\
\text { becaaaabedeca }\end{array}$ \\
\hline$y$ & $\begin{array}{l}1100111010111 \\
\text { hgcbfhgdedchh }\end{array}$
\end{tabular}

from a representative point 
Let us now consider a configuration that is not a fixed point. For $k \in$ $\{1, \ldots,\lfloor n / 2\rfloor\}$ and $k \neq n / 2$, let $E_{k}$ be the set of configurations with $k$ 1-regions, that is, $E_{k}=\left\{x \in E,|x|_{01}=k\right\}$. We show that $s_{k}=(10)^{k} 0^{n-k}$ is a representative of $E_{k}$. Let us take a configuration $x \in E_{k}$ and examine if it communicates with $s_{k}$. To show that $x \longmapsto s_{k}$, we define the following intermediary configurations (see Tab. 2):

- $x^{\prime}$ is obtained from $x$ by reducing the 1-regions to a size 1 from their right part.

- $x^{\prime \prime}$ is obtained from $x^{\prime}$ by "stacking" the 1-regions.

We now show that: $x \longmapsto x^{\prime} \longmapsto x^{\prime \prime} \longmapsto s_{k}$. We advise the readers to follow the text below with an eye on Table 2 .

Step (a): To see that $x \longmapsto x^{\prime}$, we only need to remark that the transition $\mathrm{G}$ $(110 \rightarrow 0)$ allows one to decrease the length of the 1-regions when this length is greater or equal than 2 .

Step (b): To show that $x^{\prime} \longmapsto x^{\prime \prime}$, let us first observe the following property. If a configuration $x$ contains the pattern 001, then the 1 can "move one cell to the left". Formally, if we have $z=w_{1} 001 w_{2}$, with $w_{1}, w_{2}$ two words of $\{0,1\}$ such that $\left|w_{1}\right|+\left|w_{2}\right|=n-3$, then $z \longmapsto z^{\prime}=w_{1} 100 w_{2}$. Indeed, as the transition $\mathrm{B}(001 \rightarrow 1)$ and $\mathrm{G}$ are active, we have $z \longmapsto w_{1} 011 w_{2} \longmapsto z^{\prime}$.

By iterating this argument, we have that if $x^{\prime}$ has the form $x=0^{i}(10)^{j} 10^{k} \mathrm{w}$ with $i, j \geq 0$ and $k>1$ then $x^{\prime} \longmapsto 0^{i}(10)^{j} 10^{k} w$. In words, this means that there are chains of successors which allow one to move a 1 to its leftmost position. Again, by considering each 1-region (from left to right) and moving the 1's to the left, we can build a chain such that $x^{\prime} \succ x^{\prime \prime}$.

Step (c): By considering the possibility of moving the 1's to the left as described above, we can show that for any configuration $x$ that is not a fixed point, $x \longmapsto \sigma(x)$ where $\sigma$ denotes the left shift. By repeating the shift operation as many times as needed, we can show that there exists a chain of successors such that $x^{\prime \prime} \longmapsto s_{k}$.

In the same way, it can be shown that $s_{k} \longmapsto y$, where $y$ is any configuration that is not a fixed point. We use the same arguments as above but follow a different path. First, we have that $s_{k} \longmapsto y^{\prime \prime}$, where $y^{\prime \prime}$ is the configuration obtained from $s_{k}$ with a given number of shifts such that the rightmost 1 of $y^{\prime \prime}$ coincides with the rightmost 1 of $s_{k}$ (see Tab. 2). Let $y^{\prime}$ be the configuration where all the ones are aligned with the rightmost 1's of $y$. Clearly, $y^{\prime \prime} \longmapsto y^{\prime}$. Finally, the relationship $y^{\prime} \longmapsto y$ can be obtained with the application of the transition B to make the 1-regions grow to the appropriate size.

As the application of the rule BG does not modify the number of regions, the sets $E_{k}$ are closed under the application of this rule, and, naturally, so are the fixed points. Consequently, by applying Lemma 1, the rule BG is recurrent.

The same conclusion holds for rules BFG and BCFG. Indeed, the presence of the active transitions $C$ and $F$ gives the chains of successors more possibilities of "movement" in the configuration space but does not change the communication classes. 
Table 3: Rule ADEH: two sequences of configurations showing that the communication between an arbitrary configuration and a representative configuration. On the right of each configuration is shown its image according to the transformation $T$ (see text for details). Times runs from top to bottom.

\begin{tabular}{|c|c|c|c|}
\hline 0110110001 & X.XX.X..XX & 0110110001 & $X . X X . X \ldots X X$ \\
\hline 0111110001 & $\mathrm{X} \ldots \mathrm{X} \ldots \mathrm{XX}$ & 0111110001 & $\mathrm{X} \ldots \mathrm{X} \ldots \mathrm{XX}$ \\
\hline 0111110101 & $\mathrm{X} \ldots \mathrm{XXXXX}$ & 0001010001 & $\mathrm{X} \ldots \mathrm{XXX} \ldots \mathrm{XX}$ \\
\hline 0111111101 & $\mathrm{X} \ldots \ldots \mathrm{xXX}$ & 0101010001 & $\mathrm{XXXXXX \ldots XX}$ \\
\hline 0111111111 & $\mathrm{X} \ldots \ldots \mathrm{X}$ & $(\ldots)$ & $(\ldots)$ \\
\hline 1111111111 & $\ldots \ldots$ & 0000000000 & \\
\hline
\end{tabular}

We now turn our attention to what is probably the most interesting rule of our study. The number of its communication classes depends on the divisibility of $n$ by 4 and in the general case the sizes of the classes of communication strongly vary depending on how much "freedom of movement" the system has.

Proposition 6. Rule 105:ADEH is recurrent. The number of its communication classes $C C$ depends on the ring size $n$ according to:

$$
C C(n)= \begin{cases}2 & \text { if } n \in 2 \mathbb{Z}+1 \\ n / 2+3 & \text { if } n \in 4 \mathbb{Z} \\ n / 2 & \text { otherwise. }\end{cases}
$$

Proof. To understand the structure of the communication graph of rule ADEH, let us introduce the transformation $T$ of the configuration from the states 0 and 1 to the binary alphabet $Q^{\prime}=\{., \mathrm{X}\}$ where $\mathrm{X}$ and . respectively denote the presence of absence of a change of state between a cell land its right neighbour. Formally, we associate to a configuration $x \in Q^{\mathcal{L}}$ a word $x^{\prime}=T(x) \in Q^{\prime \mathcal{L}}$ such that $\forall i \in \mathcal{L}, x^{\prime}(i)=$. if $x_{i}=x_{i+1}$ and $x^{\prime}(i)=\mathrm{X}$ otherwise. Note that $T$ is not injective since a configuration $x$ and its invert $\bar{x}$, that is, the configuration where the state of all cells has been inverted, have the same image. The function $T$ is not either surjective because, since the number of 0 -regions and 1-regions is equal on rings, only images with an even number of X's can be obtained (see Tab. 3). The two homogeneous fixed points $\mathbf{0}$ and $\mathbf{1}$ are mapped to the all-. configuration $\left(.{ }^{\mathcal{L}}\right)$.

Now, remark that any application of the rule ADEH amounts to transforming a pair . . into a pair XX or a pair XX into a pair .. in the image of $T$. Moreover, remark that a class of communication in the image process corresponds to one or two communication classes of the original rule ADEH. Indeed, given a communication class of the image, there are two possibilities: either any two pre-images of this set communicate or they do not communicate and form two distinct classes (see examples below). These properties of the images allow us to determine the classes of communication of the original rule more easily. An example of evolution of the rule and its image is shown on Fig. 3 and Tab. 3. Moreover, an example of the communication graph is shown on Fig. 4 for $n=6$. 
(a) Let us start with the case where $n$ is odd. Let us consider a configuration $x \notin\{\mathbf{0}, \mathbf{1}\}$, its image $T(x)$, and let us examine the regions of this image. Since $n$ is odd, it can be remarked that there exists at least one region of . or X in $T(x)$ that has an even size, otherwise we would have an even number of regions of odd size and $n$ would be even. This region can be made "to disappear" by changing the state of all its pairs. In other words, if for example $x=w_{1} \cdot \mathrm{X}^{2 k} \cdot w_{2}$ and $y=w_{1} \cdot{ }^{2 k+2} w_{2}$, where $k>0$ and $w_{1}$ and $w_{2}$ are arbitrary words, then $x$ and $y$ communicate. By iterating this process, we obtain a homogeneous configuration, that is, an all-. or all-X configuration, but this latter possibility is excluded because the images necessarily have an even number of X's. Consequently, $x$ communicates with any of the two homogeneous configurations $\mathbf{0}$ or 1 (the pre-images of all-.).

Now, consider the number of 00 pairs: by looking at the effect of the transitions $\mathrm{A}, \mathrm{D}, \mathrm{E}$ and $\mathrm{H}$, it can be seen that its parity is a conserved quantity, that is, if $y \in \operatorname{succ}(x)$, then $|x|_{00} \bmod 2=|y|_{00} \bmod 2$. This implies that the two configurations $\mathbf{0}$ and $\mathbf{1}$ do not communicate and the space of configurations can be partitioned into two communication classes, each one containing the configuration with an even or an odd number of 00 pairs, respectively.

(b) We can now consider the case where $n$ is even and write $n=2 k$. First let us examine what happens when $n$ is not a multiple of four, that is, when $k=$ $n / 2=2 j+1$ is odd. We claim that there are $k$ classes of communications, which representative images can be taken as the configurations $(.)^{n},(.)^{n-4 i}(\mathrm{X} .)^{2 i}$ and $(.)^{n-4 i}(. \mathrm{X})^{2 i}$, with $i \in\{1, \ldots, j\}$. In words, we can always reach an image with an even number of X's which alternate with .'s.

Indeed, by reasoning on the images and by looking at the 1-regions, we can see that the transformation of pairs $\mathrm{XX} \rightarrow \ldots$ makes an arbitrary configuration $x$ communicate with a configuration $x^{\prime}$ whose image $T\left(x^{\prime}\right)$ is either all-., or where all the X's are isolated. Then, if $T\left(x^{\prime}\right)$ contains two 1's which are separated by an even number of cells, then $x^{\prime}$ communicates with $x^{\prime \prime}$ such that the two 1's of $T\left(x^{\prime}\right)$ are replaced by two .'s in $T\left(x^{\prime \prime}\right)$. By iterating this argument, we find that $x^{\prime \prime}$ communicates with $y$, which is such that either all the X's of $T(y)$ are on even cells or they are all on odd cells. Last, we can see that $y$ communicates with the representative where the 1's have the same parity as in $T(y)$.

We now examine whether two representatives communicate. Let us consider the following quantities: $\mathfrak{e}$ and $\mathfrak{o}$, the number of even and odd cells with state $\mathrm{X}$ in the image. For a configuration $x \in \mathcal{E}_{n}$, we define: $\mathfrak{e}=\operatorname{card}\left\{i \in L ; i \in 2 \mathbb{Z}\right.$ and $\left.T(x)_{i}=\mathrm{X}\right\}$ and $\mathfrak{o}=\operatorname{card}\left\{i \in L ; i \in 2 \mathbb{Z}+1\right.$ and $\left.T(x)_{i}=\mathrm{X}\right\}$. It is then clear that $\mathfrak{q}=\mathfrak{e}-\mathfrak{o}$ is a conserved quantity, that is, if $y \in \operatorname{succ}(x)$, then $\mathfrak{q}(T(y))=\mathfrak{q}(T(x))$. Indeed, as the X's appear or disappear by pairs, the two functions $\mathfrak{e}$ and $\mathfrak{o}$ can only increase or decrease by one for each active update. Since all the representative have different values of $\mathfrak{q}$, they can not communicate (see Fig. 4).

The last detail we need to settle is whether each representative image corresponds to one or two communication classes. Indeed, remember that each image corresponds to two pre-images, which are conjugate. We claim that in the case where $k=n / 2$ is odd, each representative corresponds to a single communication class as its two pre-images communicate. The representative all- 


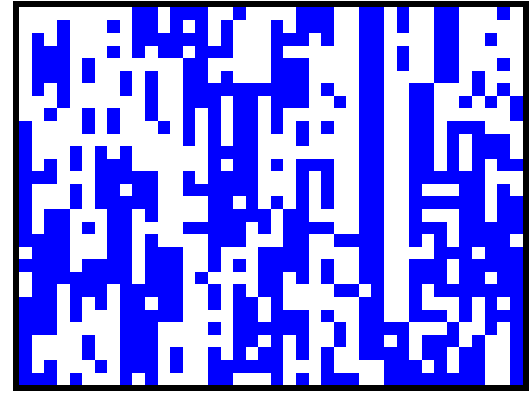

ECA 105:ADEH

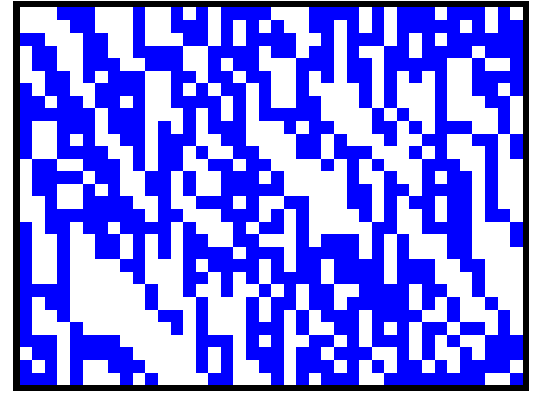

ECA 46:BDGH

Figure 3: Space-time diagrams of ECA 105: ADEH (linear number of communication classes) and ECA46:BDGH (two communication classes).

corresponds the two pre-images $\mathbf{0}$ and $\mathbf{1}$, which both communicate with $(01)^{k}$, and thus communicate together. If we take $s=(.)^{n-4 i}(\mathrm{X} .)^{2 i}$ with $i \in\{1, \ldots, j\}$ as a representative, its two pre-images are $r=0^{n-4 i}(0011)^{i}$ and its conjugate $\bar{r}=1^{n-4 i}(1100)^{i}$. To show that $r$ and $\bar{r}$ communicate, one may for example observe that $r$ communicates with $r^{\prime}=1^{n-4 i}(0011)^{i}$ (see Fig. 4). Then, by noting that $r^{\prime}$ and $\bar{r}$ have the same number of 1 's, one may construct a chain of communication from $r^{\prime}$ to $\bar{r}$ by turning pairs of 0 's into pairs of 1's (with two updates) to "shift" the pairs of 0's to their right position.

(c) We can now examine our last case: when $n$ is a multiple of four. We write $n=2 k=4 j$. In fact, all the arguments stated above still apply, with an exception which concerns the two representative images $(01)^{k}$ and $(10)^{k}$. These two images have four pre-images, the four fixed points of the rule: $(0011)^{j}$, $(0110)^{j},(1001)^{j}$ and $(1100)^{j}$. As a fixed point is its own class of communication, there are two more communication classes than in the previous case. There are thus $1+2(j-1)+4=n / 2+3$ communication classes, which respectively correspond to the following representatives: $\mathbf{0}, 0^{n-2 i}(01)^{2 i}, 0^{n-2 i}(10)^{2 i}$ with $i \in\{1, \ldots, j-1\}$, and the four fixed points.

\subsection{Rules with two communication classes}

In the following, in many cases we will prove that $\mathbf{1}$ is a representative configuration. It is thus useful to know in which case $\mathbf{1}$ will be reachable and which are the configurations that can be reached from $\mathbf{1}$.

Definition 6. For a given rule, we say that there is a density-decreasing path from $x$ to $y$ if $\exists\left(x^{0}, \ldots, x^{k}\right)$ such that $\llbracket x^{0}=x, \ldots, x^{k}=y \rrbracket$ and: $\left|x^{i+1}\right|_{1}<\left|x^{i}\right|_{1}$ for all $i \in\{0, \ldots, k-1\}$.

We say that $\mathbf{1}$ is an Olympic point of $E$ if there is a density-decreasing path from 1 to any configuration of $E \backslash \mathbf{1}$. 

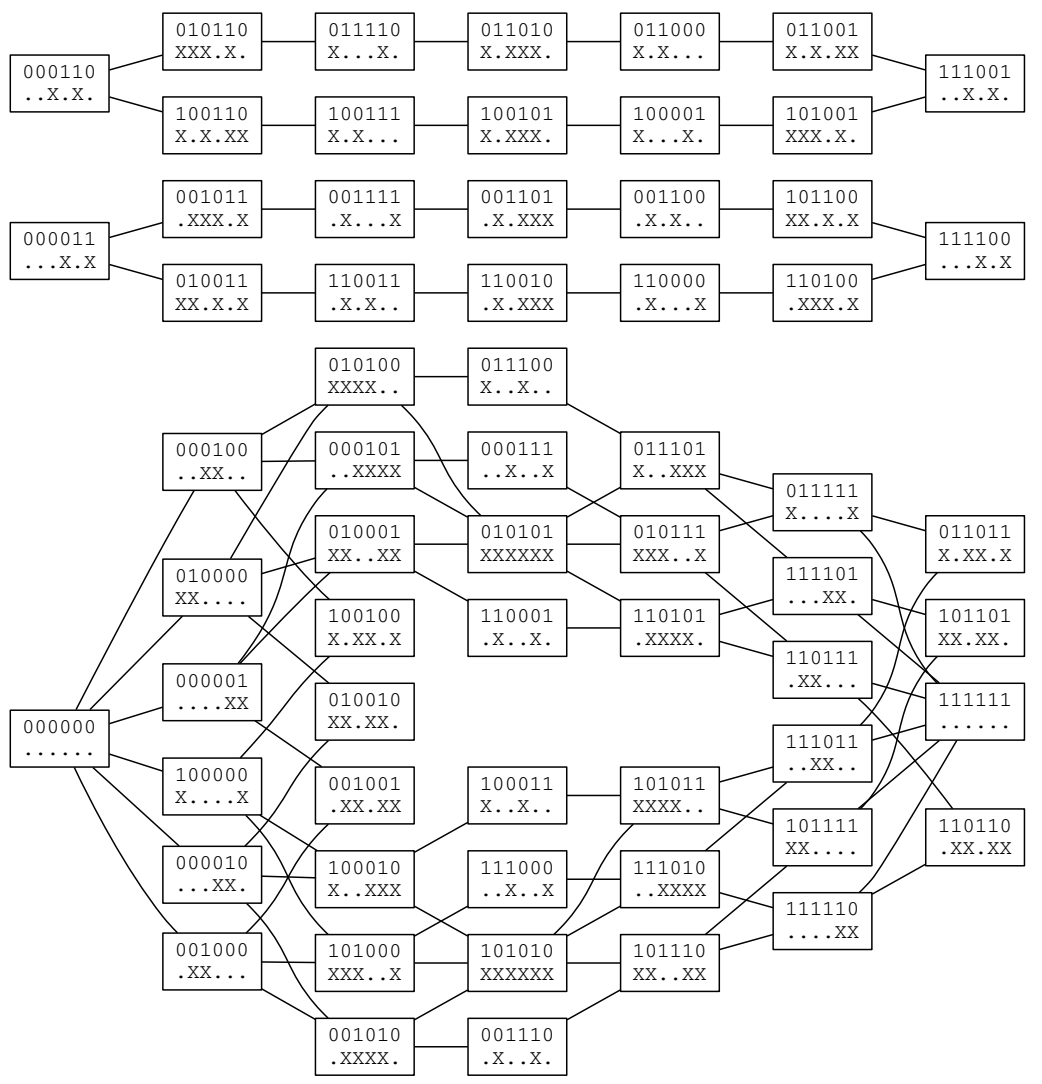

Figure 4: Communication graph for rule 105:ADEH and a ring of size $n=6$. The symbols $\mathrm{X}$ and . represent the image by a transformation which displays the differences between a cell and its right neighbour (see text for details). Selfloops are not shown, links are bidirectional. 
We say that $\mathbf{0}$ is an Avernal point of $E$ if there is a density-decreasing path from any configuration of $E \backslash \mathbf{0}$ to $\mathbf{0 .}$

It should be noted that the two definitions above are not exactly symmetric: in the first case we start from the Olympic point to reach other configurations, in the second case we start from a given configuration and reach the Avernal point $^{4}$.

Proposition 7. For a given rule, if (a) transition $\mathrm{H}$ is active and (b) transition $\mathrm{F}$ or $\mathrm{G}$ is active, then $\mathbf{1}$ is an Olympic point of $\mathcal{E}_{n} \backslash \mathbf{0}$. If conditions (a) and (b) are verified and transition $\mathrm{E}$ is active, then $\mathbf{1}$ is an Olympic point of the whole configuration space $\mathcal{E}_{n}$ and $\mathbf{0}$ is an Avernal point of $\mathcal{E}_{n}$.

Proof. The proof is straightforward. Let us assume that the conditions (a) and (b) are verified. Let us consider a configuration $x \notin\{\mathbf{0}, \mathbf{1}\}$. Then, if $x$ does not contain a pair 00, that is, if all the 0's are isolated, then, $x$ has a predecessor $y$ where a 0 has been replaced by a 1 and such that transition $\mathrm{H}$ can be applied in this cell. Formally: $\exists i \in \mathcal{L}, y \in \operatorname{succ}(x) ; \forall j \neq i, x_{j}=$ $y_{j}$ and $x_{i}=0$ and $\left(y_{i-1}, y_{i}, y_{i+1}\right)=(1,1,1)$. If $x$ contains a pair 00, as it is different from $\mathbf{0}$, it contains a triplet 001 and a triplet 100. In the same way as we did before, if transition $F$ (resp. G) is active we can construct a configuration $y$ where a 0 has been replaced by a 1 and such that transition $\mathrm{F}$ (resp. $\mathrm{G}$ ) can be applied in this cell. Formally: $\exists i \in \mathcal{L}, y \in \operatorname{succ}(x) ; x_{i}=0,\left(y_{i-1}, y_{i}, y_{i+1}\right)=$ $(0,1,1)$ or $\left(y_{i-1}, y_{i}, y_{i+1}\right)=(1,1,0)$, and $\forall j \neq i, x_{j}=y_{j}$.

As a consequence, every non-uniform configuration has a predecessor which has a higher number of 1 's, which implies that this configuration can be reached from 1.

In case $E$ is active, it can easily be seen that $\mathbf{0}$ is reachable from the configurations with only one 1 , which are themselves reachable from 1 . The uniform configuration 1 is thus an Olympic point for $\mathcal{E}_{n}$. It can also be verified that the number of 1's can always be decreased, which implies that $\mathbf{0}$ is an Avernal point.

Proposition 8. Rules 38:BDFGH, 46:BDGH and 54:BCDFGH, 60:CDGH are recurrent. Their communication classes are formed by the fixed point $\mathbf{0}$ and by the rest of the configuration space $\mathcal{E}_{n}^{*}=\mathcal{E}_{n} \backslash\{\mathbf{0}\}$.

Proof. According to Prop. 7, as transitions $\mathrm{G}$ and $\mathrm{H}$ are active, we know that $\mathbf{1}$ is an Olympic point for $\mathcal{E}_{n}^{*}$ (any configuration of this set is reachable from $\mathbf{1}$, see Tab. 4).

Now, let us take $x \in \mathcal{E}_{n}^{*}$ and show $x \longmapsto \mathbf{1}$. It can be observed that if $x \neq \mathbf{1}$, for all these rules, as either $B$ and $D$ are active or $C$ and $D$ are active, it is always possible to increase the number of 1's. Indeed, if $x$ contains the pattern 00, then it also necessarily contains the pattern 001 and 100, and transition B

\footnotetext{
${ }^{4}$ This has some logic: as expected one may go from the Olympus to any point, but the reverse is not granted, and one may also go from any point to the Avernus (the entry of the underworld in Roman mythology) but the other way round should be a more adventurous...
} 
Table 4: Rule 46:BDGH: sequence of configurations showing that the communication between an arbitrary configuration and the Olympic point $\mathbf{1}$.

\begin{tabular}{cc}
\hline$x$ & $\begin{array}{c}1100010100011 \\
\text { hgcabedecabfh }\end{array}$ \\
\hline$x^{\prime}$ & 1111010111011 \\
& hhhgdedfhgdfh \\
\hline $\mathbf{1}$ & 1111111111111 \\
& hhhhhhhhhhhhh \\
\hline
\end{tabular}

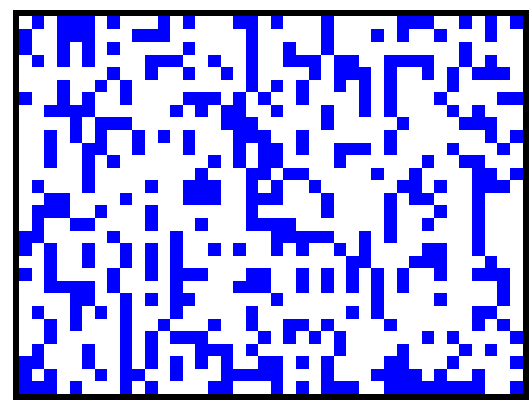

ECA 33:ADEFGH

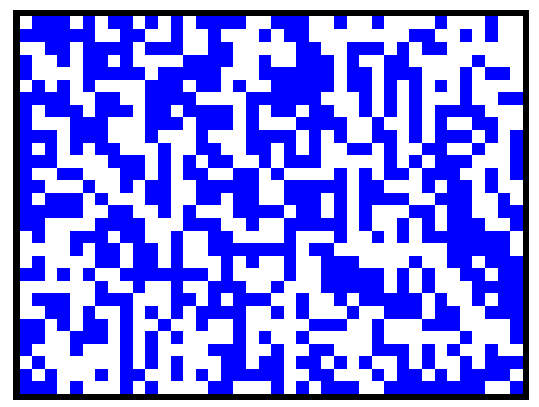

ECA 43:ABDEGH

Figure 5: Space-time diagrams of two ECA rules for which all the configurations communicate.

or $\mathrm{C}$ can be applied. If $x$ does not contains the pattern 00, this means that all the 0's are isolated, that is, in the form 101, and transition D can be applied. The configuration $\mathbf{1}$ is thus reachable from $x$ (see Tab. 4).

Consequently, $\mathbf{1}$ is a representative configuration of $\mathcal{E}_{n}^{*}$ and, given that $\mathbf{0}$ is a fixed point that can not be reached from $\mathcal{E}_{n}^{*}$, we have $\operatorname{succ}\left(\mathcal{E}_{n}^{*}\right) \subseteq \mathcal{E}_{n}^{*}$, which allows us to use Lemma 1 to conclude that the four rules are recurrent.

\subsection{Rules with a single communication class}

Proposition 9. For a ring size $n \neq 3$, the rules 33:ADEFGH and $41: A D E G H$ are recurrent. Their communication class is the whole configuration space.

The case of these two rules is interesting: its proof of recurrence is less direct than the previous rules. In particular, the rules are not recurrent for $n=3$. Indeed, if one starts from 011, the configuration 001 is reachable, but it is not possible to go back to the initial condition from this configuration as transitions $\mathrm{B}$ and $\mathrm{C}$ are inactive. The rule is however recurrent for $n>3$. 
Proof. The proof of recurrence is decomposed into three steps: (a) $\mathbf{0}$ is an Avernal point; (b) $\mathbf{1}$ is reachable from $\mathbf{0}$; (c) $\mathbf{1}$ is an Olympic point.

Steps (a) and (c) are directly obtained from Prop. 7.

To prove step (b) for the two rules, let us first assume that the ring size is even and write $n=2 k$. The sequence of configurations $\left(x_{i}\right)_{i \in\{1, \ldots, k\}}$ with $x_{i}=$ $(10)^{i} 0^{2 k-i}$ forms a chain of successors since the active transition A can always be applied in the cells in state 0 surrounded by two 0's (odd positions). We obtain $\mathbf{0} \longmapsto(01)^{k}$. Similarly, we have a chain of successors $\left(y_{i}\right)$ with $y_{i}=(11)^{i}(10) k-i$ because the transition D is active. We obtain $(01)^{k} \longmapsto \mathbf{1}$ and thus $\mathbf{0} \longrightarrow \mathbf{1}$.

Now let us consider an odd ring size $n=2 k+1$. Using the same operations as above, we can show that $\mathbf{0} \longmapsto z=1^{2 k-1} 00$ (e.g., for $z=11111100$ for $n=7$ ). Now, if we want to reach $\mathbf{1}$, it is necessary to "go backwards". With the active transition G, we have $z \longmapsto z^{\prime}=11111000$ and then, by applying transition A on the penultimate 0 , a successor $z \longmapsto z^{\prime \prime}=11111000$ with two isolated 0's (e.g., 11111010). With the transition $\mathrm{D}$, we then have $z^{\prime \prime} \longmapsto \mathbf{1}$ which gives us $\mathbf{0} \longmapsto \mathbf{1}$.

The three properties (a), (b) and (c) show the recurrence property of the two rules 33: ADEFGH and 41:ADEGH. Indeed for any two configurations $x, y \in \mathcal{E}_{n}$, we have $x \longmapsto \mathbf{0} \longmapsto \mathbf{1} \longmapsto y$.

Proposition 10. The four rules: 35:ABDEFGH, 43:ABDEGH, 51:ABCDEFGH, and 57:ACDEGH are recurrent. Their communication class is the whole configuration space.

Proof. For these three rules, by Prop. 7 we know 1 is an Olympic point. Moreover, as either the three transitions $A, B$, and D or the three transitions $A, C$, and $D$ are active, the number of 1 's can always be increased from any configuration, which implies that $\mathbf{1}$ can be reached from any configuration. It thus a representative point of the whole configuration space $\mathcal{E}_{n}$ and the rule is recurrent by Lemma 1.

\section{Discussion}

We have analysed the complete set of elementary cellular automata under fully asynchronous updating. We have asked if the behaviour of the system was recurrent and used the Markov chain theory to partition the set of rules between 46 recurrent rules (or 18 equivalence classes), and the rest of the rules, for which the return to the initial condition either never happens, or happens a finite number of times. The main tool to make this partition was to analyse the reachability relationships in the communication graph of the system. Moreover, the analysis of the communication classes showed various scaling relation of the number of communication classes as a function of the number of cells: we found the presence of an exponential number, a linear number and a constant number (two or one) or classes. The rule that displayed the greatest complexity in the analysis of the communication graph was rule 105:ADEH, which is rather surprising given that this rule can be written $f(x, y, z)=1 \oplus x \oplus y \oplus z$ (affine 
rule). It would be interesting to relate this complexity of analysis to some formal properties of the communication graph. The fact that its has fixed points of length 4 but no fixed points of smaller size may give some hints on why this rule has such a peculiar behaviour.

We can note that determining the recurrence properties of the system seems somehow an easier task than, for example, distinguishing classes of asynchronous rules as to whether they converge rapidly or not to a fixed point [Fat14]. The situation is similar with synchronous systems: only recently was achieved the classification of the 256 elementary rules according to their dynamical properties [SS12]. One may thus legitimately ask to which extent the results on the recurrence obtained above can extend to a large class of rules, radius- 2 rules or two-dimensional rules for instance.

Another path or research is to estimate the average time for returning to the initial configuration. For the "flip" rule (ECA 51), one may verify easily that the symmetry properties of the rule imply that the stationary distribution, that is, the distribution one approaches as time goes to infinity, is uniform over the set of configurations. As a result, the average return time of a $n$-sized system is $2^{n}$. This result can be generalised to the directly reversible rules: the average return time from an initial configuration is equal to the size of the communication class of this configuration. For the other rules, where a dissymmetry between the states 0 and 1 may for example create a non-uniformity between the configurations of a communication class, a more in-depth analysis would be required.

Finally, we ask what definitions of the recurrence property can be given for an infinite-size system. In this context, one may either use interacting particle systems with continuous time or $\alpha$-asynchronous systems, where the cells have a probability to be updated at each time step (see Ref. [Fat14] for more details). One may then think about defining recurrence as the ability to come back infinitely often arbitrarily close to the initial condition. However, if we take the asynchronous shift as an example, even though this rule has a tendency to make the configurations more and more uniform, it is always possible that the information that is far away influences the central zone to make it "close" to the initial configuration. In the infinite context, contrarily to what we have seen for the finite case studied here, it seems that the property to erase information locally would not be sufficient to make a rule non-recurrent. To sum up, the notion of reversibility in the context of stochastic cellular automata is a rich problem and its exploration is only at its beginning. 


\section{References}

[AP72] S. Amoroso and Y. N. Patt. Decision procedures for surjectivity and injectivity of parallel maps for tesselation structures. Journal of Computer and System Sciences, 6:448-464, 1972.

[DSS12] Sukanta Das, Anindita Sarkar, and Biplab K. Sikdar. Synthesis of reversible asynchronous cellular automata for pattern generation with specific hamming distance. In Proceedings of ACRI'12, pages 643652. Springer, 2012.

[Fat14] Nazim Fatès. A guided tour of asynchronous cellular automata. Journal of Cellular Automata, 9:387-416, 2014.

$\left[\mathrm{LPC}^{+} 16\right]$ Jia Lee, Ferdinand Peper, Sorin Dan Cotofana, Makoto Naruse, Motoichi Ohtsu, Tadashi Kawazoe, Yasuo Takahashi, Tetsuya Shimokawa, Laszlo B. Kish, and Tohru Kubota. Brownian circuits: Designs. International Journal of Unconventional Computing, 12(56):341-362, 2016.

[Mor08] Kenichi Morita. Reversible computing and cellular automata - a survey. Theoretical Computer Science, 395(1):101 - 131, 2008.

$\left[\mathrm{PLC}^{+} 13\right]$ Ferdinand Peper, Jia Lee, Josep Carmona, Jordi Cortadella, and Kenichi Morita. Brownian circuits: Fundamentals. ACM Journal on Emerging Technologies in Computing Systems, 9(1):3:1-3:24, 2013.

[Ric72] D. Richardson. Tessellations with local transformations. Journal of Computer Systems and Sciences, 6:373-388, 1972.

[SFD14] Biswanath Sethi, Nazim Fatès, and Sukanta Das. Reversibility of elementary cellular automata under fully asynchronous update. In T. V. Gopal, Manindra Agrawal, Angsheng Li, and S. Barry Cooper, editors, Proceedings of TAMC 2014, volume 8402 of Lecture Notes in Computer Science, pages 39-49. Springer, 2014.

[SMD12] Anindita Sarkar, Anindita Mukherjee, and Sukanta Das. Reversibility in asynchronous cellular automata. Complex Systems, 21(1):7184, 2012.

[SS12] Martin Schüle and Ruedi Stoop. A full computation-relevant topological dynamics classification of elementary cellular automata. Chaos, 22(4):043143, 2012.

[Tof77] Tommaso Toffoli. Computation and construction universality of reversible cellular automata. Journal of Computer Systems and Sciences, 15:213-231, 1977.

[WW13] Simon Wacker and Thomas Worsch. On completeness and decidability of phase space invertible asynchronous cellular automata. Fundamenta Informaticae, 126(2-3):157-181, 2013. 


\section{Appendix}

We calculate here an equivalent the number of communication classes of rule 105: ADEH for a rinf of size $n$. For simplicity, let us denote this number by $u_{n}=\mathcal{C}_{D H}(n)$.

This quantity verifies the following linear recurrence equation:

$$
u_{n}=u_{n-1}+u_{n-2}+u_{n-4} \text { with } u_{0}=3, u_{1}=2, u_{2}=2, u_{3}=5 .
$$

The values $u_{0}$ and $u_{1}$ are defined only for convenience and do not correspond to $\mathcal{C}_{D H}(0)$ and $\mathcal{C}_{D H}(1)$, which are excluded form our study. To solve this equation, we need to find the roots of the polynomial

$$
P(x)=x^{4}-x^{3}-x^{2}-1=(x+1)\left(x^{3}-2 x^{2}+x-1\right) .
$$

There are two real roots, $\lambda$ and -1 , and two complex roots, which are conjugate, and which we denote by $h$ and $\bar{h}$. The general solution can thus be written :

$$
u_{n}=A \lambda^{n}+B h^{n}+C \bar{h}^{n}+D(-1)^{n},
$$

where $A, B, C, D$ are four constants which belong to the set of complex numbers.

Looking at the first terms, we have:

$$
\begin{aligned}
& u_{0}=\quad A+\quad B+\quad C+D=3 \\
& u_{1}=\begin{array}{lllllll}
\lambda & A+h & B+ & \bar{h} & C & -D & =2
\end{array} \\
& u_{2}=\lambda^{2} A+h^{2} B+\bar{h}^{2} \quad C-D=2 \\
& u_{3}=\lambda^{3} A+h^{3} B+\bar{h}^{3} \quad C-D=5
\end{aligned}
$$

Now let us combine these equations with the evaluation of $q=u_{3}-2 u_{2}+u_{1}-u_{0}$. We find that $q=Q(\lambda) A+Q(h) B+Q(\bar{h}) C-(1+2+1+1) D=5-2 \times 2+2-3=0$, where $Q(x)=x^{3}-2 x^{2}+x-1$. Since by definition $Q(\lambda)=Q(h)=Q(\bar{h})=0$, we find $D=0$.

In a second step, we evaluate

$$
q^{\prime}=u_{2}-\bar{h} u_{1}=\left(\lambda^{2}-\lambda \bar{h}\right) A+\left(h^{2}-h \bar{h}\right) B=2-2 \bar{h}
$$

and

$$
q^{\prime \prime}=h\left(u_{1}-\bar{h} u_{0}\right)=(\lambda-\bar{h}) h A+h(h-\bar{h}) B=h(2-3 \bar{h}) .
$$

The evaluation of $q^{\prime}-q^{\prime \prime}$ allows one to get rid of $B$ and we have :

$$
\left(\lambda^{2}-\lambda \bar{h}-h(\lambda-\bar{h})\right) A=2-2 \bar{h}-h(2-3 \bar{h}) .
$$

It is now useful to write:

$$
\begin{aligned}
Q(x) & =x^{3}-2 x^{2}+x-1 \\
& =(x-\lambda)(x-h)(x-\bar{h}) \\
& =x^{3}-(\lambda+h+\bar{h}) x^{2}+(\lambda h+\lambda \bar{h}+h \bar{h}) x+\lambda h \bar{h}
\end{aligned}
$$


from which we obtain by identification $\lambda+h+\bar{h}=2$ and $\lambda h \bar{h}=1$.

Now, by using the relationships $h+\bar{h}=2-\lambda$ and $h \bar{h}=1 / \lambda$ in Eq. 2, we obtain: $\left(2 \lambda^{2}-2 \lambda+3\right) A=\left(2 \lambda^{2}-2 \lambda+3\right)$, which leads to $A=1$.

The algebraic or numerical estimation of the roots of $Q$ gives : $\lambda \approx 1.75488$ and $h=0.12256+0.74486 i$, whose module is strictly smaller than 1 .

We thus obtain $u_{n} \sim \lambda^{n}$, which was the desired result. 